\title{
EVALUATION OF ORAL-DENTAL HEALTH IN PATIENTS WITH PERIODONTAL PATHOLOGY AND DIABETES
}

\author{
ANDREEA DINU ${ }^{1}$, MANUELA MIHALACHE ${ }^{2}$ \\ 1,2 "Lucian Blaga” University of Sibiu
}

\begin{abstract}
Keywords:
orodental

Abstract: The present research attempts to assess the orodental health status of patients in the health, periodontal research group. The research group has been composed of 210 subjects, from both urban and rural pathology, diabetes areas, having periodontal pathologies and disorders of the glucidic metabolism. In order to obtain information about the orodental health condition as well as the orodental hygiene, we have applied the questionnaire method, this being well individualized and adapted to be relevant to the present study. Following the statistical analysis of the results, it appears that the subjects of the research group show a more pronounced periodontal pathology comparative to the control group. The study results highlight the importance of dentists' knowledge of diagnostic techniques, treatment and prevention of orodental pathologies on patients with diabetes.
\end{abstract}

\section{INTRODUCTION}

Diabetes is a common disease with simultaneous oral manifestations with impact on dental health. The term "diabetes" describes a group of disorders characterized by high blood glucose levels and abnormalities in the metabolism of carbohydrates, fats and proteins. Several oral diseases and disorders have been associated with diabetes, and periodontitis has been identified as a possible risk factor for poor metabolic control in subjects with diabetes.(1) Although bacterial infection is the main cause of periodontal disease, its progression depends on the production of inflammatory mediators, as the host's response to periodontal bacteria and their metabolic products. The hosts' reactions to bacterial invasion are different in periodontal disease and the quantity and quality of subgingival biofilm cannot explain the varied responses of the individuals. The presence of pathogenic bacteria can explain only $20 \%$ of the variability in the expression of periodontal disease. Recent studies suggest that an important part of the host response is the result of a genetic predisposition.(2)

The local, endooral conditions do not directly cause the periodontal disease, but they are factors that facilitate its onset and progression, by maintaining and supporting the accumulation of bacterial plaque. The tartar represents a pathogenic factor that facilitates the disease occurrence by maintaining the bacterial plaque in direct contact with the periodontium and by blocking the access of the dental cleansing methods. Occlusal trauma amplifies tooth mobility and widens the dentoalveolar space, contributing to the progression of the disease. Dental caries, dento-maxillary anomalies, especially those evolving with crowded dentoalveolar incongruence, parafunctions such as bruxism, vicious habits and iatrogenic factors, especially axial and transverse maladaptive prosthetic works, also favour bacterial attachment and disease progression.(3)

Chronic periodontitis can be induced by risk factors such as smoking, alcohol consumption, diet, psychological stress and depression. It has been reported that smoking is clearly involved in the onset and progression of periodontal disease and that smokers have altered qualities of gingival fluid and its components. Smoking also has a negative effect on the host's immune defence system, by inhibiting the function of neutrophils and macrophages and by affecting the defence function against periodontal bacteria.(4)

\section{AIM}

The present study aims to collect data on the orodental health status of the group of patients with diabetes mellitus and to compare these data with the witness group, represented by patients without diabetes.

\section{MATERIALS AND METHODS}

The research group contains 210 subjects who presented oro-periodontal pathology and diabetes mellitus. They came to the dental cabinet and to the University Dental Care Centre of Sibiu between March 2018 - August 2020. The study also included a witness group with the same number of subjects, but without diabetes.

The inclusion criteria for the subjects that are part of the research are:

- Presence of clinical signs of periodontal disease:

- Gingival retractions;

Gingival bleeding;

Halitosis:

Bone retraction, bone abscesses;

Dental migration or mobility;

- The existence of an OPT radiography no older than 6 months;

- Age over 25 .

Exclusion criteria from the research:

- Periodontal therapy in the last 12 months;

${ }^{1}$ Corresponding author: Andreea Dinu, Str. Irina Rosetti, Nr. 18, Cisnădie, Cod 555330, E-mail: andreea.dinu@ @ulbsibiu.ro, Phone: +40753 919643 Article received on 10.03.2021 and accepted for publication on 31.05.2021 


\section{CLINICAL ASPECTS}

- Smokers;

- Unbalanced cardiovascular diseases;

- Chronic respiratory or renal diseases;

- Osteoporosis or rheumatoid arthritis;

- Pregnant or lactating women;

- Patients with incomplete data or who refuse to participate in the study.

In order to analyse the oro-dental health condition, the subjects of the two groups who are part of the current research were asked to complete a questionnaire. The applied questionnaire is based on a standard-type questionnaire and was adapted and individualized through targeted questions about oro-dental health in order to be relevant to the present study. The questionnaire was numbered for each patient and the number remained the same for each subject in all the files within the applied methods. The questionnaire includes confidential data; therefore, the subjects' initials are used. The questions are written in a concise manner and are arranged in a logical order.

The questionnaire regarding data on the subjects' oral health contains 21 questions of which 13 have four alternatives of answers, while the rest have two alternatives. They are formulated as follows:

1. Do your gums bleed when you brush?

2. Do you clench your jaws or grind your teeth?

3. Do you have frequent toothache?

4. Do you frequently have painful lesions in the oral cavity?

5. Have you felt your teeth moving?

6. How often so you use the toothbrush?

7. How often do you use the mouthwash?

8. How often do you floss?

9. How often do you see a family doctor for a check-up?

10. How often do you see a dentist for a check-up?

11. How often do you see a dentist for scaling or brushing?

12. Do you consider having dental problems?

13. Have you undergone dental treatments?

14. Are you under dental treatment?

15. Have you undergone orthodontic treatment?

16. Have you had periodontal therapy in the last 12 months?

17. Have you had any painful spots on the skin of the face or oral mucosa?

18. Have you had prolonged bleeding after tooth extractions or other surgeries?

19. Have your teeth begun to move in recent years?

20. Do you think that these problems have worsened in recent years?

21. Do you see a dentist only when the pain occurs?

We presented the results as count and percentage. Differences between the two groups were assessed using ChiSquare or Fisher's exact test. Data analyses were performed using Core R Development Team software, Google sheets. P values $<0.05$ were considered statistically significant.(7-9)

\section{RESULTS}

Approximately half of both groups, respectively $56.67 \%$ of the control group and $52.86 \%$ of the witness group, stated they frequently have dental pain. $60 \%$ of the subjects from the control group declared they have painful lesions in the oral cavity, while only $49.05 \%$ from the witness group claim the same thing. $(\mathrm{p}=0.000)$

As for the dental mobility, $40.48 \%$ of the subjects from the control group state they have observed abnormal mobility of teeth, while $29.05 \%$ of the witness group assert that they do not have dental mobility.

From the control group, $44.76 \%$ report to the family doctor once a year for a check-up, while $50.48 \%$ from the witness group only sometimes see their doctor for a check-up $(\mathrm{p}=0.000)$

Figure no. 1. Graphic analysis of data reffering to health status resulting from the answers (group $\mathrm{C}$ or research group, group $\mathrm{M}$ or witness group)

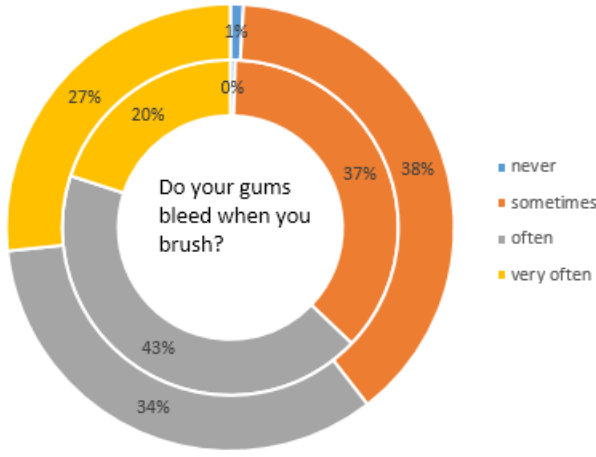

Regarding the visit at the dentist, $18.10 \%$ of the control group claim that they go for control twice or many times a year compared to those in the witness group, where $12.38 \%$ never go for a check-up. $(\mathrm{p}=0.000)$

Figure no. 2. Graphic analysis of data rreffering to health status resulting from the answers (group $\mathrm{C}$ or research group, group $\mathrm{M}$ or witness group)
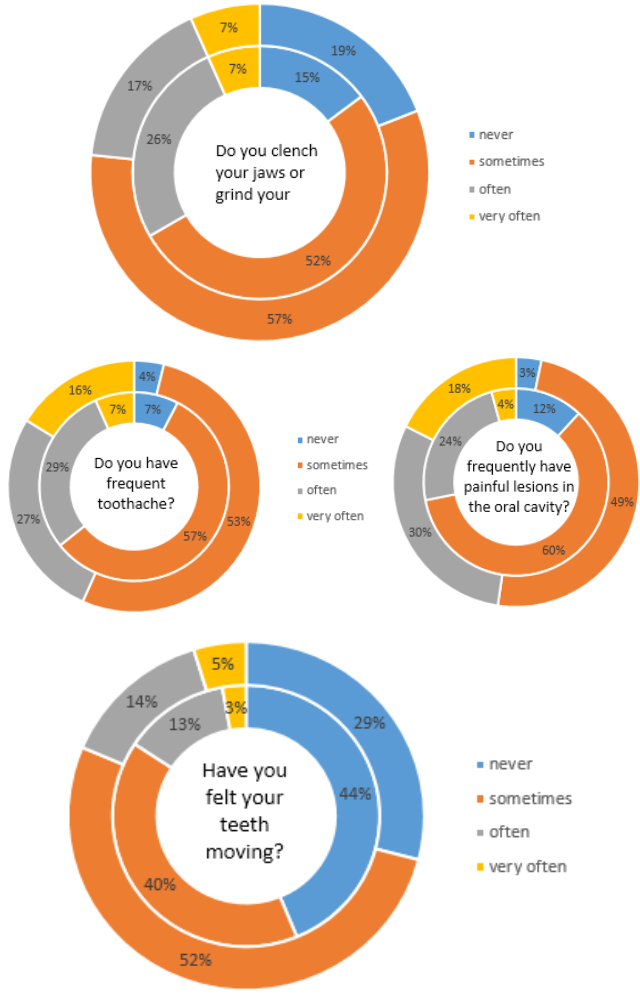

Statistically significant differences occurred following the question "How often do you use the toothbrush?" Here, $61.90 \%$ patients from the control group stated that they brush their teeth daily, as compared to the witness group, where $51.90 \%$ admitted that they sometimes brush. Also, 35.71\% subjects from the control group use the mouthwash daily, while $15.24 \%$ from the witness group never use it; $53,81 \%$ from the control group and $57.14 \%$ from the witness group sometimes floss.

From the control group, $41.43 \%$ present to the dentist's once a year for descaling, while $47.14 \%$ from the witness group only sometimes do the same thing. 


\section{CLINICAL ASPECTS}

Figure no. 3. Graphic analysis of data regarding health status following the answers to the questions (group $\mathrm{C}$ or research group, group $\mathrm{M}$ or witness group)

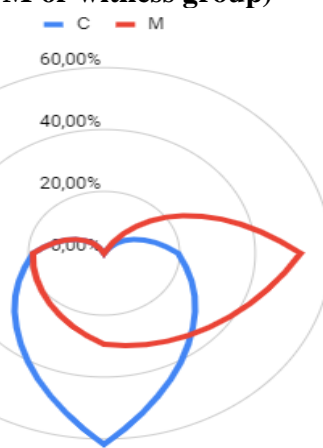

Figure no. 4. Graphic analysis of data regarding health status following the answers to the (group $\mathrm{C}$ or research group, group $\mathrm{M}$ or witness group)

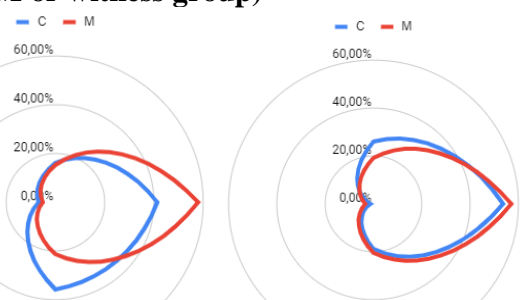

Figure no. 5. Graphic analysis of data regarding health status by answering the questions (group $\mathrm{C}$ or research group, group $\mathrm{M}$ or witness group)
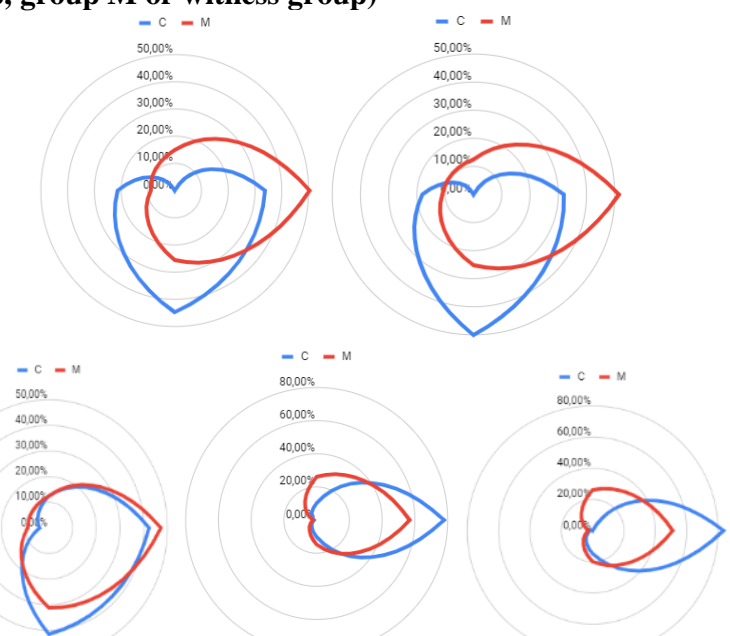

Figure no. 6. Graphic analysis of data regarding health status by answering the questions (group $\mathrm{C}$ or research group, group $M$ or witness group

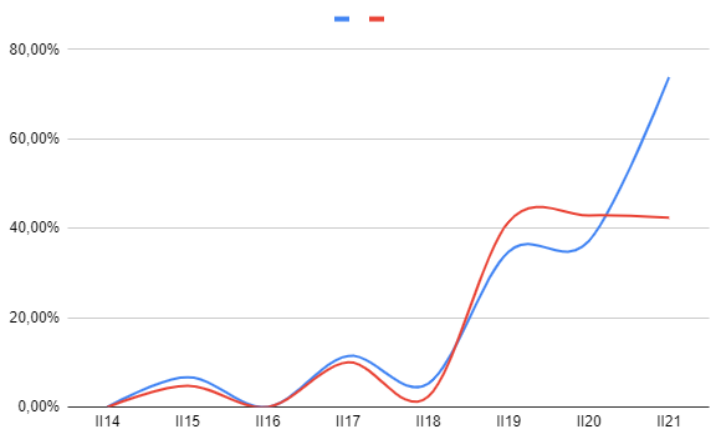

From the control group, $78.10 \%$ of the subjects consider that sometimes have dental problems, while $56.67 \%$ from the witness group state the same thing $(\mathrm{p}=0.000)$ When asked about dental treatments, $14.76 \%$ of the control group claimed that they have followed dental treatments, while $83.33 \%$ claimed that they have never followed dental treatments $(\mathrm{p}=0.000)$.

Moreover, $73.81 \%$ consider that their dental problems have worsened in the last 5 years, compared to $57.62 \%$ of the witness group who claim that the oro-dental health condition has not worsened.

\section{DISCUSSIONS}

The dentist must be acquainted to all the clinical features of diabetes mellitus, as well to its implications on oral status, in order to respond and meet the individual needs of all patients.

Most of the diabetic patients are not aware of the oral implications of hyperglycemia, hence the important role of the dentist in the constant assessment of the oral status and in the education of patients in order to reduce the oral complications of diabetes.

The prevention and management of the diabetes mellitus and the oral modifications it triggers demand continuous medical monitoring of patients, systematic control at the dentist's, and thorough daily hygiene.

The treatment of diabetic patients includes the therapy of the associated oral conditions.

\section{CONCLUSIONS}

The safe management of the diabetic patient requires effective communication between the dentist and the patient's attending physician. The dentists must be familiar with the techniques for diagnosing, treating, and preventing dental disorders in patients with diabetes.

\section{REFERENCES}

1. American DiabeteS Association. Standards of medical care in diabetes-2013. Diabetes Care. 2013;36(Suppl 1):S11-66.

2. Sandberg GE, Sundberg HE, Fjellstrom CA, Wikblad KF. Type 2 diabetes and oral health. A comparison between diabetic and non-diabetic subjects. Diabetes Res Clin Pract. 2000;50:27-34

3. Genco RJ, Sanz M. Clinical and public health implications of periodontal and systemic diseases: An overview. Periodontology. 2000;10.1111/prd.12344,83,1,(7-13).

4. Heji ES, Bukhari AA, Bahammam MA, Homida LA, Aboalshamat KT, Aldahlawi SA. Periodontal Disease as a Predictor of Undiagnosed Diabetes or Prediabetes in Dental Patients, European Journal of Dentistry, 2020.10.1055/s0040-1719208

5. Mealey, BL, Oates, TW. Diabetes mellitus and periodontal diseases.J Periodontol. 2006;77(8):1289-1303.

6. Park, OJ, Yi, H, Jeon, JH, Kang, SS, Koo, KT, Kum, KY, Chun, J, Yun, CH, Han, SH. Pyrosequencing analysis of subgingival microbiota in distinct periodontal conditions. J Dent Res. 2015;94(7):921-927.

7. R Development Team. R: A Language and Environment for Statistical Computing. R Foundation for Statistical Computing; 2009.

8. Mocan I. SPSS Introduction in data analysis; "Lucian Blaga" University publishing: Sibiu, Romania; 2005.

9. Maniu I. Data analysis techniques: statistics; "Lucian Blaga” University publishing: Sibiu, Romania; 2014. 